\title{
Kinesiophobia in rheumatoid arthritis patients: Relationship with quadriceps muscle strength, fear of falling, functional status, disease activity, and quality of life
}

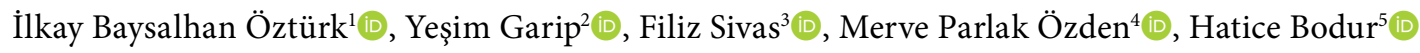 \\ ${ }^{1}$ Department of Physical Medicine and Rehabilitation, Ankara Bilkent City Hospital Physical Therapy and Rehabilitation Hospital, Ankara, Turkey \\ ${ }^{2}$ Pediatric Brain Injury Rehabilitation Clinic, Ankara Bilkent City Hospital Physical Therapy and Rehabilitation Hospital, Ankara, Turkey \\ ${ }^{3}$ Brain Injury Rehabilitation Clinic, Ankara Bilkent City Hospital Physical Therapy and Rehabilitation Hospital, Ankara, Turkey \\ ${ }^{4}$ Kahramankazan District Health Directorate, Public Health, Ankara, Turkey \\ ${ }^{5}$ Ankara Bilkent City Hospital Physical Therapy and Rehabilitation Hospital, Rheumatology, Orthopedic Rehabilitation and Pain, Ankara, Turkey
}

\begin{abstract}
Objectives: This study aims to determine the frequency of kinesiophobia in rheumatoid arthritis (RA) patients and to evaluate the relation of kinesiophobia with the knee range of motion (ROM), quadriceps muscle strength, fear of falling, functional status, disease activity, depression, and quality of life.

Patients and methods: Between September 2018 and September 2019, a total of 100 RA patients ( 25 males, 75 females; mean age: $56.1 \pm 9.3$ years; range, 32 to 69 years) and 50 healthy controls (14 males, 36 females; mean age: $54.6 \pm 9.8$ years; range, 30 to 69 years) were included. Disease activity was evaluated using the Disease Activity Score 28 (DAS28), and functional status using the Health Assessment Questionnaire (HAQ). Pain severity was measured using the Visual Analog Scale (VAS). Tampa Kinesiophobia Scale (TKS) was used to evaluate kinesiophobia. Quadriceps muscle strength and knee ROM measurements of two extremities were recorded. Depression was evaluated using the Beck Depression Inventory (BDI), fear of falling by Falls Efficacy Scale (FES) and quality of life using the Short Form-36 (SF-36).

Results: The rate of kinesiophobia was $70 \%$ in RA patients and $12 \%$ in controls, indicating a higher rate in RA patients, compared to controls (odds ratio $[O R]=44.861,95 \%$ confidence interval $[C I]: 42.571-49.052 ; p<0.05)$. This rate was $76 \%$ in females and $52 \%$ in males. Regression analysis revealed that the number of swollen and tender joints, DAS28, VAS-pain, and HAQ scores were positively associated with the TKS scores ( $p<0.05$ ). Quadriceps muscle strength and knee flexion were negatively associated with the TKS scores $(p<0.05)$. The TKS was significantly correlated with FES and BDI $(p<0.05)$. The TKS was negatively correlated with SF-36 subscales $(p<0.05)$.

Conclusion: Kinesiophobia is common in RA patients. Our study is the first to evaluate the frequency of kinesiophobia in RA patients and to show pain level, disease activity, functional status, knee flexion ROM, and quadriceps muscle strength are effective on kinesiophobia. Kinesiophobia is also associated with fear of falling and depression, negatively affecting the quality of life in terms of physical, emotional, social and mental functions. Therefore, evaluating kinesiophobia and developing targeted treatment approaches seem to be useful in increasing the quality of life in RA.

Keywords: Rheumatoid arthritis, quality of life, kinesiophobia.
\end{abstract}

Rheumatoid arthritis (RA) is a chronic, progressive autoimmune systemic disease of unknown cause, often with symmetrical involvement in small joints. ${ }^{1}$ Kinesiophobia is a type of fear-avoidance behavior defined as "fear of physical activity and movement" caused by excessive sensitivity and disturbing feeling due to painful situations or repetitive injury. ${ }^{2}$ Pain is

Received: October 26, 2020 Accepted: January 16, 2021 Published online: February 09, 2021

Correspondence: Yeşim Garip, MD. Ankara Bilkent Şehir Hastanesi Fizik Tedavi ve Rehabilitasyon Hastanesi, Pediatrik Beyin Hasarı Rehabilitasyonu, 06800 Çankaya, Ankara, Türkiye. Tel: +90 312 - 2691717 e-mail: dryesimgarip@gmail.com

\section{Citation:}

Baysalhan Öztürk I, Garip Y, Sivas F, Parlak Özden M, Bodur H. Kinesiophobia in rheumatoid arthritis patients: relationship with quadriceps muscle strength, fear of falling, functional status, disease activity, and quality of life. Arch Rheumatol 2021;36(3):427-434. 
defined as an unpleasant sensory and emotional sensation associated with real or possible tissue damage and is related to the person's past experiences. ${ }^{3}$ Fear associated with pain is accepted as a result of thoughts and negative interpretations that pain is equivalent to harmful sensory stimuli. Pain can cause physical, behavioral (escape and avoidance behavior) and cognitive (catastrophic thoughts) fear responses in the patients. ${ }^{4,5}$

Based on the fear-avoidance model, when a painful experience is interpreted as a threat by the individual, the activity would result in more pain and re-injury. The cognitive change that occurs during fear causes the perception of threat, thus causing the development of catastrophic situations such as pain, activity avoidance and disability. Pain catastrophizing, which is emphasized many times in biopsychosocial models, resulting in kinesiophobia in advanced stages, is known as focusing on pain, tendency to exaggerate pain and it is the most important psychological determinant in pain experience. Studies have suggested that kinesiophobia mainly occurs through changes in cognitive structuring such as pain-related fear and pain catastrophizing. ${ }^{6,7}$

Arthritis, joint pain, and destructive involvement in rheumatic diseases predispose individuals to develop kinesiophobia. Such a chronic painful experience can be interpreted as the equivalent of a harmful sensory stimulus, and the activity would result in more pain and re-injury. By avoiding physical activity due to pain, patients may experience not only physiological results (such as loss of muscle strength, decreased mobility), but also psychological changes, depression, and anxiety. ${ }^{8,9}$ As a result, kinesiophobia in RA can lead to impairments such as reduced mobility, muscle strength, and aerobic capacity. Persistence of this situation may cause inadequate physical functionality, disability, dissatisfaction with daily life, social isolation, depression, and poor quality of life in the long-term.

Although there are many studies in the literature examining the quality of life, pain, and depressive mood in RA, there are few studies addressing the relationship between RA and kinesiophobia. In this study, we, for the first time, aimed to determine the frequency of kinesiophobia in patients with RA and to evaluate the relation of kinesiophobia with the knee range of motion
(ROM), quadriceps muscle strength, fear of falling, functional status, depression, disease activity, and quality of life.

\section{PATIENTS AND METHODS}

This multi-center, prospective, cross-sectional study was conducted at Physical Medicine and Rehabilitation outpatient clinics between September 2018 and September 2019. A total of 100 patients with RA ( 25 males, 75 females; mean age: $56.1 \pm 9.3$ years; range, 32 to 69 years) who fulfilled the 2010 American College of Rheumatology (ACR)/European League Against Rheumatism (EULAR) classification criteria $^{10}$ and 50 age- and sex-matched healthy controls (14 males, 36 females; mean age: $54.6 \pm 9.8$ years; range, 30 to 69 years) were included in the study. Those aged 70 years, having neurological diseases, severe chronic obstructive pulmonary disease, liver and kidney failure, malignancy, uncontrolled diabetes mellitus, major psychiatric disease, pregnancy, and concomitant diseases that may cause balance problems were excluded. A written informed consent form was obtained from each participant. The study protocol was approved by the Ankara Numune Training and Research Hospital Medical Research Ethics Committee (date/no: 2018/E-18-2114). The study was conducted in accordance with the principles of the Declaration of Helsinki.

Data including age and sex of the patients, disease duration, education, occupation, marital status, and the drugs used were recorded. Additional diseases (hypertension, diabetes, peptic ulcer, thyroid dysfunction, etc.) and extraarticular involvement (osteoporosis, lung, kidney, heart, neurological, eye, etc.) were noted. In addition, the rheumatoid factor (RF) positivity, the number of swollen and tender joints, erythrocyte sedimentation rate (ESR), and C-reactive protein (CRP) levels were documented. Knee ROM measurements of all patients were made using a universal goniometer and the average values of right and left knee flexion ROM were recorded.

The quadrivariate Disease Activity Score 28 (DAS28) was used to measure RA disease activity. ${ }^{11}$ Values below 3.2 were accepted as low, values between 3.2 and 5.1 as medium, and values above 5.1 as high disease activity. 
Functional status was evaluated using the Health Assessment Questionnaire (HAQ). ${ }^{12}$ The level of pain was determined using a $10-\mathrm{cm}$ Visual Analog Scale (VAS)-pain. ${ }^{13}$

The quadriceps muscle strength was measured by manual muscle testing (MMT) according to the Medical Research Council (MRC) Scale ${ }^{14}$ and the average values of right and left quadriceps muscle strength were recorded.

Health-related quality of life was assessed using the Short Form 36 (SF-36)..$^{15}$ The Beck Depression Inventory $(\mathrm{BDI})^{16}$ was used to determine the risk for depression and to measure the severity of depressive symptoms.

The Tampa Kinesiophobia Scale (TKS) was used in the evaluation of kinesiophobia. ${ }^{17}$ The Turkish reliability and validity studies of this scale were conducted by Tunca Yilmaz et al. ${ }^{17}$ This questionnaire, which evaluates fear related to pain or previous injury, consists of 17 questions. A four-point Likert scale $(1=$ strongly disagree, $2=$ disagree, $3=$ agree, $4=$ strongly agree) is used to evaluate the answers. The minimum score is 17 and the maximum score is 68 . In this questionnaire, increased pain with movement and fear of injury, avoidance of movement due to pain are questioned. Vlaeyen et al. $^{6}$ accepted scores above 37 as the presence of kinesiophobia.

The Falls Efficacy Scale (FES) was used to evaluate the fear of falling. ${ }^{18}$ Ten questions were asked to the patients about how confident they were in performing the activities without falling. According to the answers, each question was scored between 1 and 10 (1: I trust very much; 10: I do not trust at all). Scores above 70 were accepted as the presence of fear of falling.

\section{Statistical analysis}

Study power analysis and sample size calculation were performed using the $\mathrm{G}^{*}$ Power version 3.1.9.4 software (Heinrich-Heine-Universität Düsseldorf, Düsseldorf, Germany). In the analysis ( $\alpha=0.05)$ (tails=2) performed to have the power of the study at least 0.80 , at least 96 and 48 (a total of 144) patients were required in the sample groups.

Statistical analysis was performed using the IBM SPSS for Windows version 20.0 software (IBM Corp., Armonk, NY, USA). Descriptive data were expressed in mean \pm standard deviation (SD), median (min-max) or number and frequency. The Kruskal-Wallis test was used to examine whether the numerical data was normally distributed. Differences between the groups were evaluated using the independent samples t-test. The Chi-square test was used to compare groups of categorical variables. Logistic regression analysis was performed to identify the factors affecting the level of kinesiophobia. The relationship between continuous variables was evaluated using the Pearson's correlation analysis. A $p$ value of $<0.05$ was considered statistically significant at $95 \%$ confidence interval (CI).

\section{RESULTS}

Baseline demographic and clinical characteristics of the patients and controls are shown in Table 1. There was no significant difference between the mean age of the patients and the controls ( $p>0.05)$. For extra-articular involvement in RA patients, 11 (11\%) patients had pulmonary involvement and one (1\%) patients had renal involvement. Of the patients, 88 (88\%) had no extra-articular involvement. The RF positivity was found in 78 (78\%) of the patients.

The TKS scores above 37 were accepted as the presence of kinesiophobia, and the rate of kinesiophobia was found to be $70 \%$ in the RA patients and $12 \%$ in the controls. The rate of kinesiophobia was significantly higher in RA patients, compared to the control group (odds ratio $[\mathrm{OR}]=44.861$, 95\% CI: 42.571-49.052; $\mathrm{p}<0.05$ ) (Table 2). According to sex, the TKS score was $>37$ in 57 women (76\%) and 13 men (52\%), indicating a statistically significant difference between the two sexes $(p<0.05)$.

The mean kinesiophobia score assessed by TKS in the patient group with RA was $41.0 \pm 10.3$ (range, 20.0 to 58.9). In the control group, it was $26.7 \pm 8.5$ (range, 19.0 to 47.0 ). The mean TKS score in the RA patients was found to be significantly higher than the control group $(p<0.05)$. In addition, the mean FES score was significantly higher in the patients with RA $(\mathrm{p}<0.05)$ (Table 3).

In the regression analysis, the mean number of swollen and tender joints, DAS28, VAS-pain, and 
Table 1. Demographic and clinical characteristics of study population

\begin{tabular}{|c|c|c|c|}
\hline & Mean \pm SD & Median & Min-Max \\
\hline Age (year) & $56.1 \pm 9.3$ & 58.00 & $32.00-69.00$ \\
\hline Disease duration (year) & $15.8 \pm 8.5$ & 16.00 & $1.00-35.00$ \\
\hline Number of swollen joints & $0.4 \pm 1.2$ & 0.00 & $0.00-10.00$ \\
\hline Number of tender joints & $4.7 \pm 7.2$ & 0.00 & $0.00-25.00$ \\
\hline Erythrocyte sedimentation rate & $21.1 \pm 13.7$ & 19.50 & $2.00-73.00$ \\
\hline C-reactive protein & $8.7 \pm 9.8$ & 5.00 & $0.05-48.00$ \\
\hline Disease Activity Score 28 & $2.8 \pm 1.2$ & 2.54 & $0.50-5.78$ \\
\hline Visual Analog Scale-pain & $4.2 \pm 3,2$ & 5.00 & $0.00-10.00$ \\
\hline Health assessment questionnaire & $0.4 \pm 0.5$ & 0.15 & $0.00-2.30$ \\
\hline Quadriceps muscle strength (mean of right and left) & $5.0 \pm 0.4$ & 5.00 & $3.00-5.00$ \\
\hline Knee flexion ROM (mean of right and left) & $119.1 \pm 12.9$ & 120.00 & $80.00-140.00$ \\
\hline Tampa Kinesiophobia Scale & $41.0 \pm 10.3$ & 41.50 & $20.00-58.00$ \\
\hline Falls efficacy scale & $19.8 \pm 15.3$ & 12.00 & $10.00-91.00$ \\
\hline SF-36-physical function & $61.1 \pm 30.1$ & 65.00 & $0.00-10.00$ \\
\hline SF-36-bodily pain & $63.0 \pm 27.4$ & 67.50 & $10.00-100.0$ \\
\hline SF-36-physical role & $49.3 \pm 46.7$ & 50.00 & $0.00-100.0$ \\
\hline SF-36-vitality & $49.7 \pm 23.2$ & 55.00 & $0.00-85.00$ \\
\hline SF-36-emotional role & $58.0 \pm 41.5$ & 66.70 & $0.00-100.0$ \\
\hline SF-36-social function & $82.1 \pm 23.7$ & 100.00 & $0.00-100.0$ \\
\hline SF-36-mental health & $60.0 \pm 20.6$ & 58.00 & $8.00-96.00$ \\
\hline SF-36-general health & $43.3 \pm 21.2$ & 40.00 & $0.00-95.00$ \\
\hline Beck depression inventory & $11.0 \pm 7.8$ & 9.00 & $0.00-32.00$ \\
\hline
\end{tabular}

SD: Standard deviation; Min: Minimum; Max: Maximum; ROM: Range of motion; SF-36: Short Form 36.

Table 2. Frequency of kinesiophobia in patients with rheumatoid arthritis

\begin{tabular}{|c|c|c|c|c|}
\hline Kinesiophobia (TKS>37) & $\mathrm{n}$ & $\%$ & Difference $(95 \% \mathrm{CI})$ & $p$ \\
\hline RA patients $(n=100)$ & 70 & 70 & \multirow{2}{*}{$44.861(42.571-49.052)$} & \multirow{2}{*}{$<0.00001$} \\
\hline Controls $(n=50)$ & 6 & 12 & & \\
\hline
\end{tabular}

TKS: Tampa Kinesiophobia Scale; CI: Confidence interval; RA: Rheumatoid arthritis; " $\mathrm{p}<0.05$ (significant).

Table 3. Comparison of TKS and FES Scores of study population

\begin{tabular}{lccccccc}
\hline & \multicolumn{2}{c}{ RA patients $(\mathrm{n}=100)$} & & \multicolumn{2}{c}{ Controls $(\mathrm{n}=50)$} & \\
\cline { 2 - 3 } & Mean \pm SD & Median & & Mean \pm SD & Median & $p$ \\
\hline TKS & $41.0 \pm 10.3$ & 41.5 & & $26.7 \pm 8.5$ & 24.5 & $<0.00001^{*}$ \\
FES & $19.8 \pm 15.3$ & 12.00 & & $12.8 \pm 3.8$ & 11.5 & $0.002^{*}$ \\
\hline
\end{tabular}

TKS: Tampa Kinesiophobia Scale; FES: Falls Efficacy Scale, RA: Rheumatoid arthritis; SD: Standard deviation; * $p<0.05$ (significant). 
Table 4. Effects of clinical variables on TKS in rheumatoid arthritis patients

\begin{tabular}{|c|c|c|}
\hline Clinical variables & B & $p$ \\
\hline Age & -0.003 & 0.971 \\
\hline Disease duration & 0.033 & 0.699 \\
\hline Swollen joints & 0.187 & $0.035^{*}$ \\
\hline Tender joints & 0.356 & $0.032^{*}$ \\
\hline VAS-pain & 0.184 & $0.048^{*}$ \\
\hline DAS28 & 0.655 & $0.008^{*}$ \\
\hline HAQ & 0.249 & $0.029 *$ \\
\hline $\begin{array}{l}\text { Quadriceps muscle strength } \\
\text { (mean of right and left) }\end{array}$ & -0.185 & $0.047^{*}$ \\
\hline Knee flexion ROM (mean of right and left) & -0.237 & $0.007^{*}$ \\
\hline
\end{tabular}

HAQ were positively associated with TKS scores. The quadriceps muscle strength and knee flexion were negatively associated with the TKS scores $(p<0.05)$. The coefficient and corrected $R 2$ values are given in Table 4.

The mean TKS scores were significantly correlated with FES and BDI scores $(p<0.05)$. The TKS was negatively correlated with SF-36 subscales. Considering the correlation coefficients, the highest negative correlation was between TKS and SF-36 physical function subscale $(\mathrm{r}=-0.632)$. The SF-36 physical function subscale was followed by SF-36 energy and SF-36 physical role limitation, respectively $(\mathrm{r}=-0.610$ and $r=-0.572$, respectively) (Table 5).

\section{DISCUSSION}

In the current study, we investigated the frequency and level of kinesiophobia in patients with RA and to evaluate the relation of kinesiophobia with ROM, quadriceps muscle strength, fear of falling, functional status, depression, pain, disease activity and quality of life. In our study, the frequency of kinesiophobia in RA patients was found to be 70\%. In the control group, this rate was $12 \%$ and the difference was statistically significant. Our study is the first to evaluate the frequency of kinesiophobia in RA patients.
Table 5. Correlation of TKS with other clinical variables

\begin{tabular}{lcc}
\hline Clinical variables & $p$ & $R$ \\
\hline FES & $0.0001^{*}$ & 0.523 \\
BDS & $0.0001^{*}$ & 0.601 \\
SF-36-physical function & $0.0001^{*}$ & -0.632 \\
SF-36-bodily pain & $0.0001^{*}$ & -0.522 \\
SF-36-physical role & $0.0001^{*}$ & -0.572 \\
SF-36-vitality & $0.0001^{*}$ & -0.610 \\
SF-36-emotional role & $0.0001^{*}$ & -0.464 \\
SF-36-social function & $0.0001^{*}$ & -0.396 \\
SF-36-mental health & $0.0001^{*}$ & -0.467 \\
SF-36-general health & $0.0001^{*}$ & -0.547 \\
\hline
\end{tabular}

TKS: Tampa Kinesiophobia Scale; FES: Falls Efficacy Scale; BDI: Beck Depression Inventory; SF-36: Short Form 36, ${ }^{*} p<0.05$ (significant).

In a study by Oskay et al. ${ }^{19}$ conducted in Turkish patients with ankylosing spondylitis, the frequency of kinesiophobia was reported as $66.6 \%$. Bränström et al. $^{20}$ also found the frequency of kinesiophobia as 56\% in Swedish patients with musculoskeletal pain. In another study conducted in Sweden, the frequency of kinesiophobia was reported to be $54 \%$ in the patients aged between 18 and 65 years who applied to the physical therapy center with musculoskeletal pain. ${ }^{21}$ In these two studies, the cut-off value was taken as $>37$, similar to our study. Lundberg et al. ${ }^{21}$ reported that this phenomenon should be taken into consideration in clinical situations, since kinesiophobia has a negative effect on rehabilitation results.

In our study, the female-to-male ratio was $3: 1$, consistent with the sex ratio in RA. ${ }^{1}$ When the patients with RA were evaluated according to sex, $76 \%$ of women and $52 \%$ of men had kinesiophobia. The difference between men and women was statistically significant. Similarly, in the study of Verbunt et al. ${ }^{22}$ conducted in Norwegian patients with chronic low back pain, the kinesiophobia level in female patients was found to be higher than that of male patients. Contrary to the findings in these studies, in the study of Bränström et al., ${ }^{20}$ the rate of kinesiophobia was higher in males (72\%) than females (48\%) with musculoskeletal pain. 
In our study, the mean kinesiophobia score evaluated with TKS was 41, indicating a significantly higher score than the control group. Review of the literature reveals that our study is the third study to evaluate the kinesiophobia level in RA. Previously, Lööf et al. ${ }^{8}$ evaluated kinesiophobia in the patients with RA using the modified Fear-Avoidance Beliefs Questionnaire and found a high level of kinesiophobia in the patients. Similarly, Kınıkl et al. ${ }^{23}$ classified RA patients as those receiving conventional and biological disease-modifying antirheumatic drugs and combined therapy in their study and found the TKS scores to be 43.31, 42.21, and 38.11, respectively. These results are consistent with our findings.

In the present study, age and duration of disease were not associated with TKS scores in RA patients. In the study of Kinikl et al., ${ }^{23}$ age was found to be positively correlated with TKS. Similar to our study, no relationship was recorded between disease duration and TKS in the aforementioned study. Our patient group consisted of the patients with low disease activity, regular follow-up, and regular medication use which may have led to the finding that TKS was associated with disease activity, but not with age or disease duration. In their study, Vlaeyen et al. ${ }^{6}$ found a negative correlation between the fear of movement and disease duration and attributed this to the fact that the fear of movement in patients might decrease during the chronicity of the disease. According to Vlaeyen et al., ${ }^{6}$ avoiding daily activities in acute painful situations is an adaptive reaction that develops spontaneously in patients and affects the healing process positively. However, one of the reasons for the continuation of avoidance behavior in patients with chronic pain is not only the short-term effect of reduced suffering, but also the effect of certain beliefs and expectations. All these support the lack of effect of disease duration on TKS.

In our study, the effect of clinical variables on TKS in RA patients was evaluated and pain was found to be positively associated with the TKS scores. On the basis of fear-avoidance model, pain is interpreted as a threat by the individual and may generate an idea that the activity would cause more pain and re-injury. The cognitive change that occurs during fear creates a perception of threat, thus leading to the development of catastrophic behaviors such as pain, activity avoidance, and disability. Several studies have demonstrated that kinesiophobia is mainly caused by factors that cause changes in cognitive structure such as painrelated fear and pain catastrophizing. Similar results were reported in the previous studies in the literature..$^{8,23}$ Löof et al. $^{8}$ drew attention to the presence of pain-related avoidance behavior in RA patients and emphasized that these patients should be given cognitive behavioral therapy. A similar relationship was found in the patients with ankylosing spondylitis, ${ }^{19}$ chronic low back pain, ${ }^{24,25}$ and systemic lupus erythematosus. ${ }^{26}$ Based on these findings, pain is one of the most common symptoms which predisposes to kinesiophobia in RA patients. In these patients, effective pain relief should be provided by using both pharmacological treatments and physical modalities, and the patients should be encouraged to make exercises which are appropriate for their general condition, age, and cultural and environmental factors.

Another finding in our study is that the numbers of swollen and tender joints and DAS28 scores were positively associated with TKS scores. Joint swelling and tenderness cause a high disease activity and pain, leading to catastrophic behaviors such as activity avoidance. To the best of our knowledge, our study is the first to reveal the relationship between disease activity and kinesiophobia in patients with RA.

Furthermore, in our study, we found that quadriceps muscle strength and knee flexion angle were negatively associated with the TKS scores in patients with RA. Again, our study is the first to reveal this relationship in RA patients. This is a finding, we expected, as well. The negative relationship between kinesiophobia and quadriceps muscle strength has been previously reported by Özmen et al. ${ }^{27}$ in patients with knee osteoarthritis. In another study, the knee flexion ROM was negatively correlated with kinesiophobia among female patients with patellofemoral pain. ${ }^{28}$

Another remarkable finding in RA patients is the effect of functional disability on kinesiophobia. The relationship between disability and kinesiophobia in RA patients has been previously reported by Lööf et $\mathrm{al}^{8}$ and Kınıklı et al. ${ }^{23}$ Based on these findings, 
it should be considered that disability may cause kinesiophobia and that kinesiophobia may increase the existing functional limitations.

Although the relationship between depression and kinesiophobia has been examined in previous studies, our study is the first to demonstrate this relationship in patients with RA. Expectedly, the patients' fear of movement and avoidance behavior are associated with psychosocial factors. Oskay et al. ${ }^{19}$ reported that BDI scores were correlated with TKS values in patients with ankylosing spondylitis. In another study conducted in Turkey, the relationship between kinesiophobia and depression was evaluated in the patients with systemic lupus erythematosus, and TKS was found to be significantly correlated to BDI. ${ }^{26}$ In another study conducted in Brazil, the relationship between $\mathrm{BDI}$ and TKS in the patients with chronic low back pain was studied, and higher kinesiophobia was reported in the patients with depression. ${ }^{29}$

Another finding of the current study was the presence of a positive correlation between kinesiophobia and fear of falling. To the best of our knowledge, our study is the first study to delineate this relationship in patients with RA. Previously, this relationship was only shown in the patients with Parkinson's disease. It is an expected result that those who have a fear of falling would also have a fear of moving. ${ }^{30}$

Based on our findings, kinesiophobia negatively affects the quality of life in the context of physical function, pain, energy, emotional, social and mental functions. A negative correlation was found between all subscales of SF-36 and TKS. The same relationship was previously reported by Lööf et al. $^{8}$ in RA patients and by Bağlan Yentür et al. ${ }^{26}$ in patients with systemic lupus erythematosus. Taken together, we conclude that evaluating kinesiophobia in RA and preventing it with targeted multidisciplinary approaches, such as cognitive therapies, would be effective in improving the quality of life.

Nonetheless, there are some limitations to the present study. First, our study is a cross-sectional study and, therefore, the cause-effect relationship was unable to be determined exactly. Second, the scales used to evaluate depression, quality of life, and kinesiophobia are mainly based on the patients' reports. Further studies are needed to confirm these findings.
In conclusion, early diagnosis and treatment in RA patients with kinesiophobia are of utmost importance to halt the disease progression and, consequently, the preserve the future functional status of the patient. It is important to develop kinesiophobia prevention strategies to identify RA patients at a higher risk of kinesiophobia at an early stage and to provide them to participate in the physical activity. As soon as the diagnosis is made, medical treatment should be initiated and necessary information should be given about the benefits of physical activity and the problems that may arise with inactivity. For this purpose, it would be appropriate to hold training meetings for kinesiophobia and to give information forms to patients. We believe that group therapies can be effective on kinesiophobia, as well.

\section{Declaration of conflicting interests}

The authors declared no conflicts of interest with respect to the authorship and/or publication of this article.

\section{Funding}

The authors received no financial support for the research and/or authorship of this article.

\section{REFERENCES}

1. Scutellari PN, Orzincolo C. Rheumatoid arthritis: sequences. Eur J Radiol 1998;27 Suppl 1:S31-8.

2. Knapik A, Saulicz E, Gnat R. Kinesiophobia introducing a new diagnostic tool. J Hum Kinet 2011;28:25-31.

3. Treede RD. The International Association for the Study of Pain definition of pain: as valid in 2018 as in 1979 , but in need of regularly updated footnotes. Pain Rep 2018;3:e643.

4. Turk DC, Wilson HD. Fear of pain as a prognostic factor in chronic pain: conceptual models, assessment, and treatment implications. Curr Pain Headache Rep 2010;14:88-95.

5. Edwards RR, Cahalan C, Mensing G, Smith M, Haythornthwaite JA. Pain, catastrophizing, and depression in the rheumatic diseases. Nat Rev Rheumatol 2011;7:216-24.

6. Vlaeyen JW, Kole-Snijders AM, Rotteveel AM, Ruesink $\mathrm{R}$, Heuts PH. The role of fear of movement/(re)injury in pain disability. J Occup Rehabil 1995;5:235-52.

7. Lethem J, Slade PD, Troup JD, Bentley G. Outline of a Fear-Avoidance Model of exaggerated pain perception--I. Behav Res Ther 1983;21:401-8.

8. Lööf H, Demmelmaier I, Henriksson EW, Lindblad S, Nordgren B, Opava $\mathrm{CH}$, et al. Fear-avoidance beliefs 
about physical activity in adults with rheumatoid arthritis. Scand J Rheumatol 2015;44:93-9.

9. Verhoeven F, Tordi N, Prati C, Demougeot C, Mougin F, Wendling D. Physical activity in patients with rheumatoid arthritis. Joint Bone Spine 2016;83:265-70.

10. Aletaha D, Neogi T, Silman AJ, Funovits J, Felson DT, Bingham CO 3rd, et al. 2010 Rheumatoid arthritis classification criteria: an American College of Rheumatology/European League Against Rheumatism collaborative initiative. Arthritis Rheum 2010;62:2569-81.

11. Prevoo ML, van 't Hof MA, Kuper $\mathrm{HH}$, van Leeuwen MA, van de Putte LB, van Riel PL. Modified disease activity scores that include twenty-eight-joint counts. Development and validation in a prospective longitudinal study of patients with rheumatoid arthritis. Arthritis Rheum 1995;38:44-8.

12. Küçükdeveci AA, Sahin $H$, Ataman $S$, Griffiths $B$, Tennant A. Issues in cross-cultural validity: example from the adaptation, reliability, and validity testing of a Turkish version of the Stanford Health Assessment Questionnaire. Arthritis Rheum 2004;51:14-9.

13. Price DD, McGrath PA, Rafii A, Buckingham B. The validation of visual analogue scales as ratio scale measures for chronic and experimental pain. Pain 1983;17:45-56.

14. Medical Research Council. Aids to the examination of the peripheral nervous system. London: HMSO; 1981. p. 1.

15. Bilir Kaya B, İçaasıoğlu A. Reliability and validity of the Turkish version of short form 36 (SF-36) in patients with rheumatoid arthritis. J Surg Med 2018;2:11-6.

16. Hisli N. Beck depresyon envanterinin üniversite öğrencileri için geçerliği, güvenirliği. Türk Psikoloji Dergisi;1988;6:3-13.

17. Tunca Yllmaz Ö, Yakut Y, Uygur F, Uluğ N. Tampa Kinezyofobi Ölçeği'nin Türkçe versiyonu ve test-tekrar test güvenirliği. Fizyoter Rehabil 2011;22:44-9.

18. Morgan MT, Friscia LA, Whitney SL, Furman JM, Sparto PJ. Reliability and validity of the Falls Efficacy Scale-International (FES-I) in individuals with dizziness and imbalance. Otol Neurotol 2013;34:1104-8.

19. Oskay D, Tuna Z, Düzgün İ, Elbasan B, Yakut Y, Tufan A. Relationship between kinesiophobia and pain, quality of life, functional status, disease activity, mobility, and depression in patients with ankylosing spondylitis. Turk $\mathrm{J}$ Med Sci 2017;47:1340-7.

20. Bränström H, Fahlström M. Kinesiophobia in patients with chronic musculoskeletal pain: differences between men and women. J Rehabil Med 2008;40:375-80.

21. Lundberg $M$, Larsson $M$, Ostlund $H$, Styf J. Kinesiophobia among patients with musculoskeletal pain in primary healthcare. $\mathrm{J}$ Rehabil Med 2006;38:37-43.

22. Verbunt JA, Seelen HA, Vlaeyen JW, van der Heijden GJ, Knottnerus JA. Fear of injury and physical deconditioning in patients with chronic low back pain. Arch Phys Med Rehabil 2003;84:1227-32.

23. Kınıklı Gİ, Güney Deniz H, Karahan S, Ateş A, Turgay M, Kınıkl G. Predictors of Fear of Movement in Patients with Rheumatoid Arthritis. Turk J Physiother Rehabil 2018;29:11-7.

24. Altuğ F, Ünal A, Kilavuz G, Kavlak E, Çitişli V, Cavlak U. Investigation of the relationship between kinesiophobia, physical activity level and quality of life in patients with chronic low back pain1. J Back Musculoskelet Rehabil 2016;29:527-31.

25. Marshall PWM, Schabrun S, Knox MF. Physical activity and the mediating effect of fear, depression, anxiety, and catastrophizing on pain related disability in people with chronic low back pain. PLoS One 2017;12:e0180788.

26. Bağlan Yentür S, Karatay S, Oskay D, Tufan A, Küçük H, Haznedaroğlu Ş. Kinesiophobia and related factors in systemic lupus erythematosus patients. Turk J Med Sci 2019;49:1324-31.

27. Özmen T, Gafuroğlu Ü, Altun Güvenir A, Ziraman İ, Özkurt B. Relationship between kinesiophobia, quadriceps muscle strength and quality of life in patients with knee osteoarthritis. Turkish Journal of Geriatrics 2017;20:38-45.

28. Silva Dde O, Briani RV, Pazzinatto MF, Ferrari D, Aragão FA, Azevedo FM. Reduced knee flexion is a possible cause of increased loading rates in individuals with patellofemoral pain. Clin Biomech (Bristol, Avon) 2015;30:971-5.

29. Antunes RS, de Macedo BG, Amaral Tda S, Gomes Hde A, Pereira LS, Rocha FL. Pain, kinesiophobia and quality of life in chronic low back pain and depression. Acta Ortop Bras 2013;21:27-9.

30. Monticone M, Ferrante S, Ambrosini E, Rocca B, Secci C, Foti C. Development of the Tampa Scale of Kinesiophobia for Parkinson's disease: confirmatory factor analysis, reliability, validity and sensitivity to change. Int J Rehabil Res 2015;38:113-20. 